fell from 3,900 before treatment to $2,600 / \mathrm{mm}^{3}$ while on methyldopa with a drop in neutrophils from 61 to $54 \%$ and a relative increase in lymphocytes from 35 to $42 \%$. While on debrisoquine the white cell count increased to $8,100 / \mathrm{mm}^{3}$ and $65 \%$ of this was neutrophils and $30 \%$ lymphocytes. Further study of this patient is necessary to decide if this is a toxic effect of methyldopa.

\section{Discussion}

In this trial of debrisoquine and methyldopa in the treatment of patients with moderately severe hypertension, neither drug showed a superior ability to lower the supine and standing diastolic blood pressure with a minimum of side effects. Methyldopa, however, produced a significant reduction in both the supine and the standing systolic blood pressure which was not influenced by the diuretic supplement.

Side effects which are inevitable with all the sympathetic blocking agents can be a reason for patients defaulting. Of the 38 patients, two were unable to tolerate methyldopa because of side effects. This intolerance of side effects with methyldopa has been noted previously in up to $20 \%$ of patients (Prichard et al., 1968).

It is very difficult to compare side effects, and to facilitate this they were classified as major or minor. No significant difference in the incidence of the major and minor side effects with either drug was found in those who completed both phases of the trial, though the type of side effect varied with each drug. This also came out in the patient's reasons for preferring one drug to the other, but no clue was apparent from which a patient's choice of drug could have been deduced in advance. Tiredness was the most characteristic side effect with methyldopa, patients often not realizing how tired they were until they changed drugs. Postural and exer- cise hypotension were more prominent with debrisoquine, though they were easily controlled by a small adjustment in dosage. This prominence of different side effects with one sympathetic blocking agent more than another is a manifestation of their slightly different pharmacological actions at the cellular level. Detailed statistical analysis confirmed that debrisoquine produced greater orthostatic hypotension than $\varnothing$ methyldopa, and this was not influenced by the addition of $५$. hydrochlorothiazide $\mathrm{K}$ (see Chart). This latter finding is surprising because of our previous impression (Heffernan and Carty, 1970) and that of Gent and Bacon (1967) that the postural effect of debrisoquine is potentiated by diuretics.

Another reason not often considered why patients who require long-term drug treatment for any disease default is the expense of the drugs. The cost of the average daily dose of debrisoquine used in this trial is about one-third that of methyldopa.

We are grateful to Professor T. B. Counihan for allowing us to study patients under his care and for his continuing interest in the trial. Dr. D. L. Scott, of Roche Products Ltd., and Dr. M. F. Grayson, of Merck Sharp \& Dohme, deserve our thanks for helping in the design of the trial, for supplying the tablets, and for always being available to discuss problems and progress. Professor G. J. Bourke, of the department of social and preventive medicine, University College, Dublin, and Mr. K. Wilson, of the Economic and Social Research Institute, Dublin, gave invaluable help in the statistical analysis for which we are grateful.

\section{References}

Gent, A. E., and Bacon, A. P. C. (1967). Practitioner, 198, 673 Heffernan, A. G. A., and Carty, A. T. (1970). Irish fournal of Medical Science, 3, 37.

Prichard, B. N. C., Johnston, A. W., Hill, I. D., and Rosenheim, M. L. (1968). British Medical fournal, 1, 135.

Rose, G. A., Holland, W. W., and Crowley, E. A. (1964). Lancet, 1, 296.

Skinner, C., Coull, D. C., and Johnston, A. W. (1969). Lancet, 2, 564.

\title{
Lipoprotein Electrophoretic Patterns, Serum Lipids, and Coronary Heart Disease
}

\author{
J. R. MASAREI, MARGARET SUMMERS, D. H. CURNOW, K. J. CULLEN, \\ M. G. MCCALL， N. S. STENHOUSE， T. A. WELBORN
}

British Medical fournal, 1971, 1, 78-82

\section{Summary}

Lipoprotein electrophoresis was performed on serum from subjects with and without coronary heart disease, and the patterns compared with the serum concentrations of triglyceride and cholesterol. The beta- and pre-beta-lipoproteins, expressed as a percentage of the total lipoprotein, correlate strongly with

Royal Perth Hospital, Western Australia

J. R. MASAREI, M.D., M.C.P.A., Registrar in Biochemistry

MARGARET SUMMERS, B.SC., Chemist, Department of Biochemistry

University of Western Australia

D. H. CURNOW, PH.D., F.R.A.C.I., Professor of Clinical Biochemistry M. G. MCCALL, D.PHIL., M.R.A.C.P., Senior Lecturer in Medicine N. S. STENHOUSE, M.sC., Director of Raine Medical Statistics Unit T. A. WELBORN, M.B., M.R.C.P., Raine Research Fellow

Georgiana Molloy Centre, Busselton, Western Australia K. J. CULLEN, M.D., M.R.C.P.ED., General Practitioner the cholesterol and triglyceride concentrations, respectively. The beta- and pre-beta-lipoprotein concentrations are even more strongly correlated with these lipid measurements. The lipoprotein pattern does not have greater discriminant value for coronary heart disease than does the triglyceride or cholesterol concentration. There would seem to be little justification for the use of lipoprotein electrophoresis in screening the general population for coronary heart disease.

\section{Introduction}

Following the 1966 Busselton community health survey (Curnow et al., 1969) a follow-up study was made in July 1968 of a group of subjects with a survey diagnosis of "probable" or "possible" coronary heart disease together with a group of controls. The aspect of the study reported here concerned the proposition that lipoprotein patterns might prove to be a valuable index of the presence of coronary heart disease, thus adding to the power of biochemical determinations to locate subjects at risk of this disease. 
Fredrickson et al. (1967) suggested a classification of the hyperlipidaemias which has been widely accepted. Lipoprotein electrophoresis forms an integral part of this classification. Electrophoresis of lipoproteins differentiates high serum triglyceride levels due to chylomicrons (exogenous triglyceride) from those due to endogenous triglyceride (pre-beta). It has been shown that lipoprotein fractions characteristically carry different proportions of cholesterol, triglyceride, and phospholipid (Ewing et al., 1965). One would therefore expect a correlation between the total concentration of a lipid class in the serum and the amount of lipoprotein with which it is predominantly associated. Such correlations have been shown by Mitchell et al. (1966) with respect to cholesterol, and by Allard and Goulet (1967) with respect to cholesterol and glyceride, using an immunological method for the determination of beta-lipoprotein.

Lipoprotein electrophoresis has been performed most commonly on paper by the method of Lees and Hatch (1963). Though cellulose acetate has been suggested as a suitable medium it has not been generally accepted. Chin and Blankenhorn (1968) suggested the use of cellulose acetate membrane (Sepraphore III, Gelman Co.) and found clear separations, including that of the pre-beta fraction from the beta. We have found that satisfactory separations can be obtained by using the Microzone apparatus (Beckman Co.) and Sepraphore III cellulose acetate membrane. In this study we examined the relationship between the serum concentrations of cholesterol and triglyceride and the lipoprotein fractions as separated electrophoretically. The results were expressed as a percentage of the total lipoprotein in each class. We attempted to ascribe to the pre-beta and beta fractions an absolute value by determining on each serum the total low-density linonrotein bv a turbidimetric method (Walton and Scott, 1964) from which the amount of lipoprotein in the pre-beta and beta fractions could be determined. Finally, the values were examined in relation to the presence or absence of coronary heart disease.

\section{Subjects}

The subjects consisted of individuals who at the 1966 Busselton survey fulfilled the criteria for a diagnosis of "probable" or "suspect" coronary heart disease together with controls matched for age and sex. The diagnosis of probable or suspect coronary heart disease was made on the basis of electrocardiographic findings and a positive history for probable or suspect angina pectoris or myocardial infarction. In addition to the standard questionnaire (Rose and Blackburn, 1968) the subiects were asked, "Did you see your doctor because of this pain or discomfort?" If the answer was affirmative the subiect was then asked, "What did he say it was?" Those subjects giving positive angina pectoris histories on the standard Rose questionnaire who gave their doctor's diagnosis as angina or heart disease or acceptable svnonym were classified as having probable angina pectoris. Those giving a positive history for myocardial infarction on the standard Rose questionnaire who gave their doctor's diagnosis as coronary occlusion or heart attack or myocardial infarction were classified as probable myocardial infarction. The remaining subjects classified as suspect angina pectoris or suspect myocardial infarction had fulfilled the required criteria of the Rose questionnaire but had not been seen by their doctor, or the doctor had stated a non-cardiac diagnosis.

The probable coronary heart disease group comprised those individuals with a history of probable angina pectoris and/or pronounced $\mathrm{Q}$-wave changes, complete heart block, complete left bundle-branch block (Minnesora codes 1.1 or $1.2,6.1,7 \cdot 1$ ) (Blackburn et al., 1960), other subjects with pronounced S-T depression and/or frank $T$-wave inversion but without tall $\mathbf{R}$ waves (Minnesota codes $4.1,5.1$, or 5.2 , but without 3.1 ), and all subjects with a positive history for probable myocardial infarction alone not already mentioned above.

The suspect coronary heart disease group comprised those subjects with a history of suspect angina pectoris or myocardial infarction and/or borderline electrocardiographic abnormalities of $Q$ wave, $S-T$ segment, and $T$ wave (codes $1.3,4.2$ or $4.3,5.3$ ), excluding tall $R$ waves and/or complete right bundle-branch block and/or auricular fibrillation-that is, excluding codes $3.1,7.2,8.5$ respectively.

Subjects were classified as having probable or suspect coronary heart disease on both the 1966 and 1968 surveys. A combined classification was used here designed to class as having probable coronary heart disease those subjects who had probable coronary heart disease on either survey; those not included who had suspect coronary heart disease on either survey were given that classification for this study, and the remainder were classified as normal or "no coronary heart disease." Lipoprotein electrophoresis was performed on serum from 103 subjects ( 52 men and 51 women) taken at random. The age composition of this population is shown in Figs. 1 and 2 . It is to be noted that the age distribution of
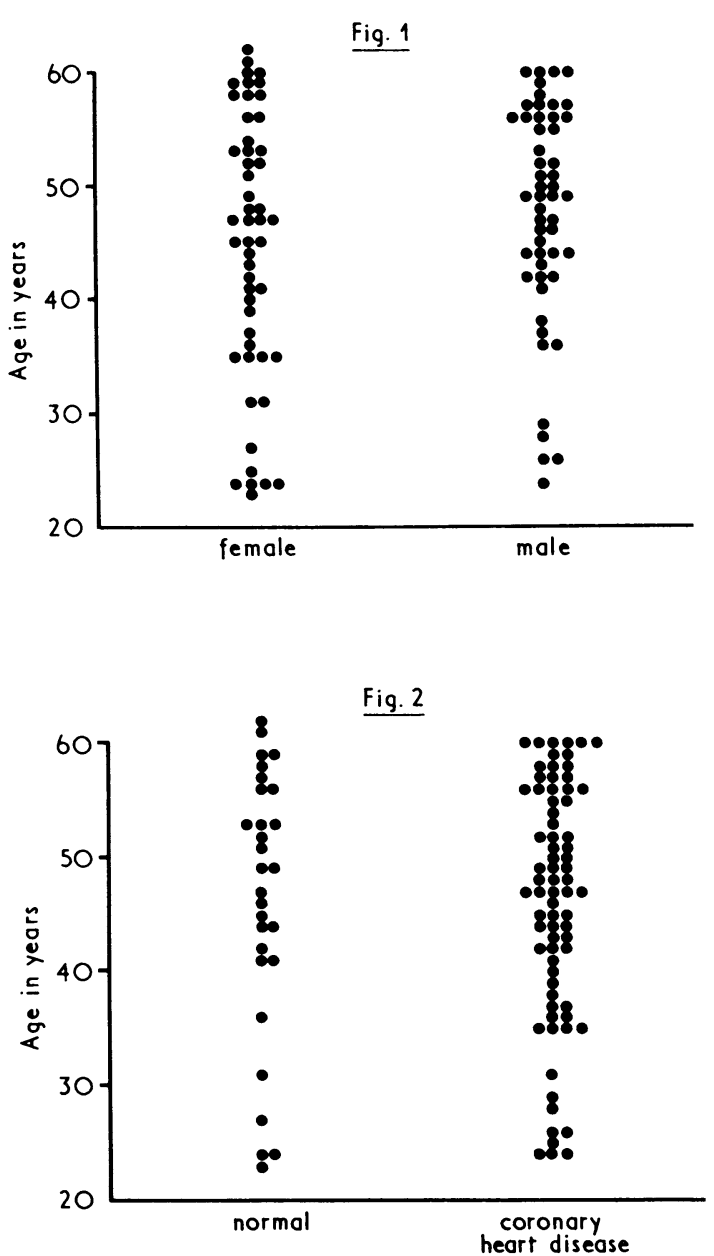

FIG. 1-Distribution of subjects with respect to age and sex. FIG. 2-Distribution of subjects in coronary heart disease groups with respect to age.

the normal and combined suspect and probable coronary heart disease groups is similar. Taking the intervals 21-39, $40-49$, and 50 years and over, $\chi^{2}=0.308, P>0.5$. Similarly, taking cut-off points at 52 and 57 years the age distributions are found to be similar, and it is concluded that the coronary heart disase and normal groups do not differ with respect to age. 


\section{Methods}

Blood was collected after an overnight fast. Lipoprotein electrophoresis was performed within 24 hours. The conditions were as follows: constant voltage (240); barbitone buffer pH 8.6, 0.075 M; duration 40 minutes. Samples were applied with the Microzone applicator, which delivers about $0.25 \mu \mathrm{l}$. After electrophoresis the strips were placed in an oil red $\mathrm{O}$ stain prepared by the method of Chin and Blankenhorn (1968) and incubated at $37^{\circ} \mathrm{C}$ overnight. They were then washed in tapwater for 10 minutes and cleared in glycerin. The result was a strip that was translucent, but with a pale red background. For scanning they were placed between glass slides, the edges sealed with adhesive tape and then scanned in the JoyceLoebl chromoscan (slit 5010). The areas under the peaks were integrated by the machine and the relative areas expressed as a percentage of the total.

Low-density lipoproteins were measured by the method of Walton and Scott (1964), in which low-density lipoproteins are precipitated by dextran sulphate in the presence of calcium ion at $\mathrm{pH}$ 9.0. Low-density lipoprotein as measured in this method is equivalent to the beta- and pre-beta electrophoretic fractions. Though the results are expressed in $\mathrm{mg} / 100 \mathrm{ml}$ of serum the standard curve obtained with the reagent supplied by the manufacturer of the materials used (B.D.H. serum low-density lipoproteins kit) was not tested for accuracy. We view these figures as having significance only fro the derivation of a figure for the beta- and pre-betalipoprotein concentrations.

Cholesterol was determined on the AutoAnalyzer (N24a methodology) and triglyceride by the semi-automated method of Lofland (1964).

\section{Results}

The percentage of the total lipoprotein (mean \pm S.D.) found in the three fractions was as follows: Alpha 27.0 \pm 11.6; beta $54.5 \pm 10.6$; pre-beta $18.4 \pm 10.2$.

\section{INTERRELATIONSHIPS}

There is a highly significant positive correlation between the pre-beta-lipoprotein percentage and the triglyceride concentration ( $r=0.69, P<0.001$ ) (Fig. 3). There is also a highly significant positive correlation between beta percentage and cholesterol concentration ( $r=0.33, P<0 . \mathrm{CO1})$ (Fig. 4).

The pre-beta-lipoprotein concentration was calculated from the expression:

\section{Pre-beta percentage}

Pre-beta percentage + beta percentage

Total low-density lipoprotein

By expressing the pre-beta-lipoprotein in these terms the correlation between it and the triglyceride concentration has increased to 0.83 (Fig. 5).

The beta-lipoprotein concentration was derived from the following expression:

\section{$\frac{\text { Beta percentage }}{\text { Total low-density }}$}

Again, by expressing the beta-lipoprotein in these terms the correlation coefficient with respect to cholesterol has increased to 0.81 (Fig. 6).

Effect of Age and Sex.-The cholesterol, triglyceride, and pre-beta-lipoprotein concentrations increase significantly with age $(r=0.23,0.21$, and 0.23 respectively; $P<0.05$ in each case). There were no significant differences in any variable between the sexes.

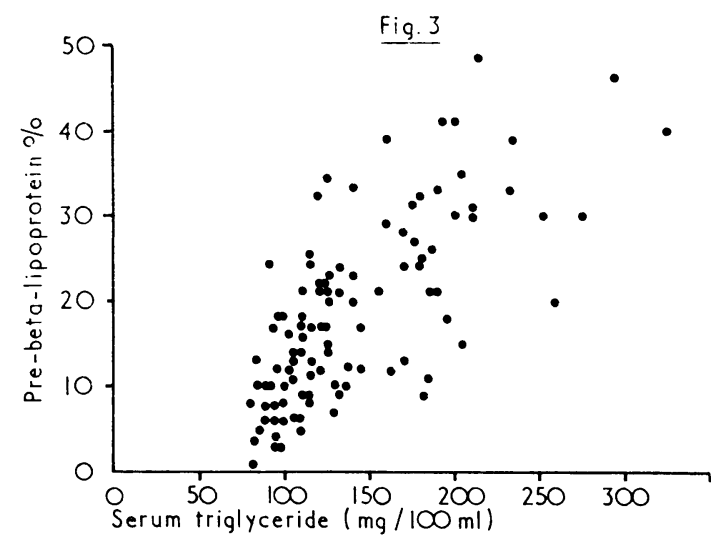

要

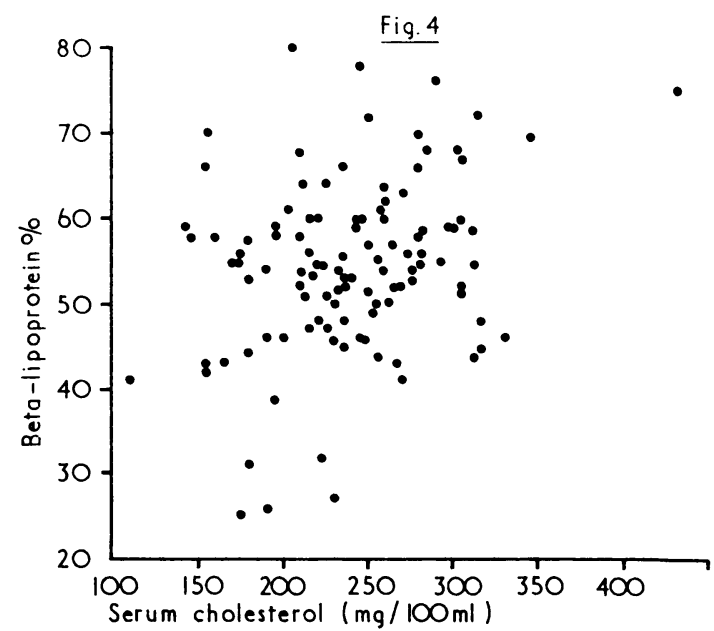

FIG. 3-Relationship between the percentage of total lipoprotein present in the pre-beta fraction and the serum triglyceride concentration $(r=0.69 ; \mathrm{P}<0.001)$. FIG. 4Relationship between the percentage of total lipoprotein present in the beta fraction and the serum cholesterol concentration $(r=0.33 ; P<0.001)$.

Effect of Body Weight.-When expressed as a percentage of desirable weight body weight was found to correlate significantly with the pre-beta percentage and the pre-beta-lipoprotein concentration (Table $I$ ) at the $P<0.001$ level of significance, and with the beta-lipoprotein concentration at the $\mathbf{P}<0.05$ level. With respect to the triglyceride and cholesterol concentrations the correlations were significant at the $\mathrm{P}<0.001$ and $\mathrm{P}<0.01$ levels respectively. The correlations with the unadjusted body weight were similar except that for N cholesterol the level of significance was $P<0.05$. The correlations with blood sugar of unadjusted weight and percentage desirable weight were significant at the $\mathrm{P}<0.01$ level. There was no significant correlation with the coronary heart disease group.

Lipid Values and Coronary Heart Disease.-None of the lipid or lipoprotein measurements was significantly correlated with the coronary heart disease groups. The discriminant value of these determinations, however, was tested at various cut-off points. To do this the normal and suspect groups were combined and compared with the "probable" group, in

TABLE I-Correlation of Percentage Desirable Weight with Other Variables Measured

\begin{tabular}{|c|c|c|c|c|c|}
\hline Variable & & & & & Significance \\
\hline $\begin{array}{l}\text { Pre-beta percentage } \\
\text { Pre-beta-lipoprotein concentration } \\
\text { Beta percentage .. } \\
\text { Beta-lipoprotein concentration } \\
\text { Triglyceride concentration } \\
\text { Cholesterol concentration } \\
\text { Fasting blood sugar }\end{array}$ & $\begin{array}{l}\cdots \\
\cdots \\
\cdots \\
\cdots \\
\cdots\end{array}$ & $\begin{array}{l}\cdots \\
\therefore \\
\cdots \\
\cdots \\
\cdots \\
\cdots\end{array}$ & $\begin{array}{l}\cdots \\
\cdots \\
\cdots \\
\cdots \\
\cdots \\
\cdots\end{array}$ & $\begin{array}{l}0.406 \\
0.496 \\
0.077 \\
0.234 \\
0.374 \\
0.265 \\
0.306 \\
0 \cdot 112\end{array}$ & $\begin{array}{l}P<0.001 \\
P<0.001 \\
\quad N . S . \\
P<0.05 \\
P<0.001 \\
P<0.01 \\
P<0.01 \\
\quad \text { N.S. }\end{array}$ \\
\hline
\end{tabular}



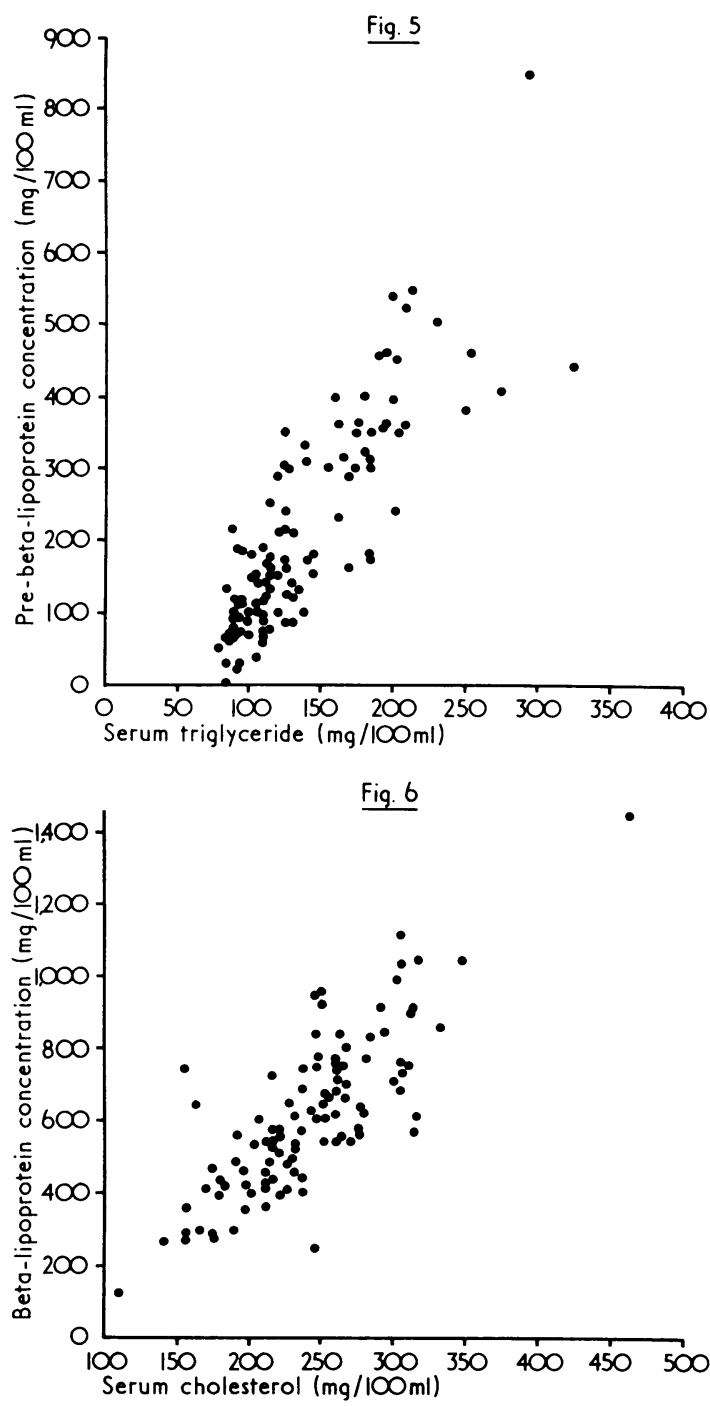

FIG. 5-Relationship between the serum triglyceride concentration and the pre-beta-lipoprotein concentration $(r=0.83 ; P<0.001)$. FIG. 6-Relationship between the serum cholesterol concentration and the beta-lipoprotein concentration $(r=0.81 ; P<0.001)$.

whom the diagnosis of coronary heart disease could be accepted with some certainty. In this respect triglycerides gave highly significant discrimination at values of 126 and $150 \mathrm{mg} / 100 \mathrm{ml}$ (67th and 80th percentiles respectively) $\left(\chi^{2}=7.5, \mathrm{P}<0.05\right.$, and $12.32, \mathrm{P}<0.001$, respectively). For the pre-beta-lipoprotein concentration no discriminant value was found-for example, $200 \mathrm{mg} / 100 \mathrm{ml}$, the 67 th percentile for the "normal" group, $\chi^{2}=2 \cdot 1, P<0.05$. Cholesterol and betalipoprotein concentration were similarly of no value. In the whole Busselton coronary follow-up study (Welborn et al., $1969 \mathrm{a}, 1969 \mathrm{~b})$ triglyceride was found to have greater discriminant value than cholesterol. The distribution of values for triglyceride and pre-beta-lipoprotein concentrations in the coronary heart disease groups is shown in Figs. 7 and 8.

Some of the correlations discussed below are summarized in Table II.

\section{Discussion}

This study has shown that a pre-beta-lipoprotein fraction can be recognized on electrophoresis of serum from most subjects, whether normal or having probable coronary heart disease, in age groups over 21 years. It has been stated (Smith, 1957; Fredrickson et al., 1967) on the basis of electrophoresis on
TABLE II-Correlation Coefficients between the Lipoproteins and Other Values Determined

\begin{tabular}{|c|c|c|c|c|c|}
\hline & $\begin{array}{c}\text { Alpha } \\
\%\end{array}$ & $\begin{array}{c}\text { Beta } \\
\%\end{array}$ & $\begin{array}{c}\text { Pre-beta } \\
\%\end{array}$ & $\begin{array}{c}\text { Beta- } \\
\text { lipoprotein } \\
\text { Concentration }\end{array}$ & $\begin{array}{c}\text { Pre-beta- } \\
\text { lipoprotein } \\
\text { Concentration }\end{array}$ \\
\hline 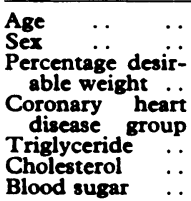 & $\begin{array}{r}-0.118 * \\
0.269 \ddagger \\
-0.292 \ddagger \\
-0.064 * \\
-0.348 \\
-0.296 \ddagger \\
-0.240 \ddagger\end{array}$ & $\begin{array}{r}-0.060^{*} \\
-0.164^{*} \\
0.055^{*} \\
0.002^{*} \\
-0.290^{*} \\
0.3295^{*} \\
-0.087^{*}\end{array}$ & $\begin{array}{r}0.190^{\circ} \\
-0.132 \\
0.4405 \\
0.067 * \\
0.6915 \\
0.000 \\
0.3645\end{array}$ & $\begin{array}{r}0.150^{*} \\
-0.028 * \\
0.251 \dagger \\
-0.050^{*} \\
0.270 \ddagger \\
0.809 \\
0.040^{*}\end{array}$ & $\begin{array}{r}0.231 \dagger \\
-0.042^{4} \\
0.4675 \\
0.024 \\
0.8305 \\
0.275 \ddagger \\
0.3895\end{array}$ \\
\hline
\end{tabular}

* Not significant. $+P<0.05$. $\$ P<0.01$. $\quad$ S $P<0.001$.

paper that a pre-beta band is usually absent in normal people. It is possible that paper is not the optimal medium for this separation. Noble (1968) separated lipoproteins on agarose-gel and also reported that a pre-beta fraction is always detected in serum from adults.

It has been confirmed in this study that the electrophoretic beta-lipoprotein is strongly correlated with the serum cholesterol level and the pre-beta with serum triglyceride. It is recognized that this method for quantitating lipoproteins electrophoretically has limitations, associated with dye uptake
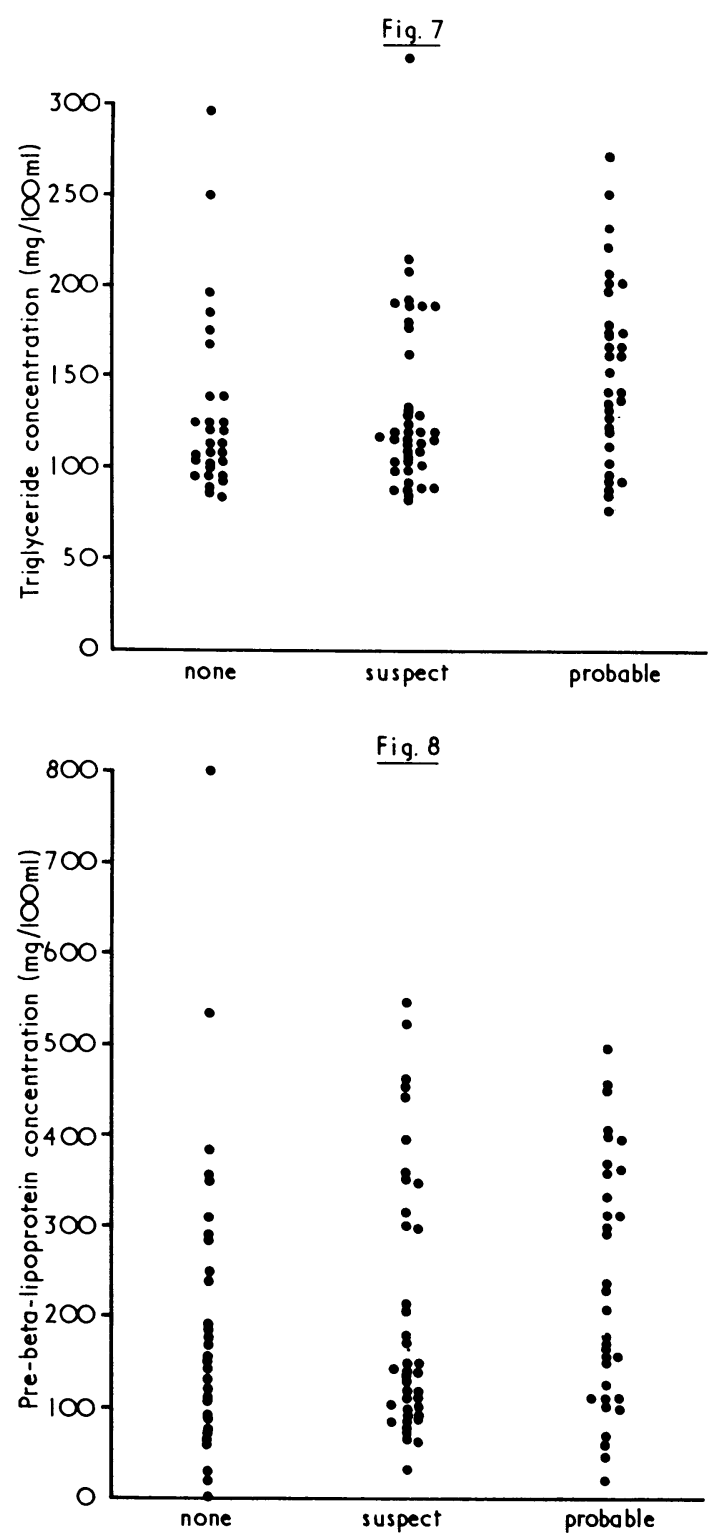

FIG. 7-Triglyceride concentration in the three coronary heart disease groups. FIG. 8-Pre-beta-lipoprotein concentration in the three coronary heart disease groups. 
and other factors. Nevertheless, it has shown these associations with the lipid concentrations and would seem to give similar information to these lipid measurements. These strong correlations between the lipoprotein values and the triglyceride and cholesterol concentrations were reflected in their common correlations with other factors examined-for example, body weight, fasting blood sugar, and age.

An interesting observation is the association of body weight and fasting blood sugar with triglycerides and pre-betalipoproteins (Tables I and II). The data are consistent with the recognized trend towards obesity and impaired glucose tolerance found in Fredrickson's type IV hyperlipoproteinaemia (increased endogenous triglyceride with raised pre-betalipoprotein) (Fredrickson et al., 1967).

Lipoprotein electrophoresis may be of value in the identification of a raised triglyceride as being due to chylomicrons or endogenous triglyceride, or being due to the abnormal very-low-density lipoprotej,ns with beta mobility ("broad beta," Fredrickson's type III), but in terms of discriminating subjects having coronary heart disease from those without, the determination of the lipoprotein pattern does not have any value over the determination of triglyceride and cholesterol. Furthermore, it has the disadvantages of not being amenable at present to automation and requiring greater time and effort in its performance.

Ostrander et al. (1967) examined the lipoprotein electrophoretic patterns in subjects with and without coronary heart disease and found that the presence of a pre-beta-lipoprotein did not discriminate between the groups. The study of Hatch et al. (1966) of a more thoroughly diagnosed group of young men with and without coronary heart disease suggested that the pre-beta-lipoprotein band was significantly correlated with the presence of coronary heart disease. We would conclude that lipoprotein electrophoresis need not be used in screening of the general population directed towards the detection of coronary heart disease.

We wish to thank Mrs. Clare Johnstone for assistance with the W statistical analysis. We acknowledge the financial assistance of the Bank of New South Wales; the Royal Perth Hospital Research @ Fund; the University of Western Australia; the National Heart $c$ Foundation of Australia; Boehringer Ingelheim Pty. Ltd.; Geigy Australia Pty. Ltd.; Australian Hoechst Ltd.; and Mr. F. Clayton, Busselton.

\section{References}

Allard, C., and Goulet, C. (1967). Canadian Medical Association fournal, 97,1321 .

Blackburn, H., Keys, A., Simonson, E., Rautaharju, P., and Punsar, S. (1960). Circulation, 21, 1160.

Chin, H. P., and Blankenhorn, D. H. (1968). Clinica Chimica Acta, 20, 305. Curnow, D. H., Cullen, K. J., McCall, M. G., Stenhouse, N. S., and Welborn, T. A. (1969). Australian fournal of Science, 31, 281.

Ewing, A. M., Freeman, N. K., and Lindgren, F. T. (1965). Advances in Lipid Research, 3, 25.

Lipid Research, 3, 25.
Fredrickson, D. S., Levy, R. I., and Lees, R. S. (1967). New England

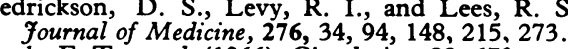

Hatch, F. T., et al. (1966). Circulation, 33, 679.

Lees, R. S., and Hatch, F. T. (1963). Fournal of Laboratory and Clinical Medicine, 61, 518.

Lofland, H. B. (1964.) Analytical Biochemistry, 9, 393

Mitchell, F. L., Pearson, J., and Strauss, W. T. (1966). Clinica Chimica Acta, 14,1 .

Noble, R. P. (1968). Fournal of Lipid Research, 9, 693.

Ostrander, L. D., Neff, B. J., Block, W. D., Francis, T., and Epstein, F. H. 6 (1967). Annals of Internal Medicine, 67, 34

Rose, G. A., and Blackburn, H. (1968). Cardiovascular Survey Methods. Geneva, World Health Organization.

Smith, E. B. (1957). Lancet, 2, 910

Walton, K. W., and Scott, P. J. (1964). Fournal of Clinical Pathology, 17,627 .

Welborn, T. A., et al., (1969a). American fournal of Epidemiology, 89, 521.

Welborn, T. A., et al. (1969b). Second International Symposium on . Atherosclerosis, Chicago. In press.

\title{
High Altitude and House-dust Mites
}

\author{
F. TH. M. SPIEKSMA， P. ZUIDEMA， M. J. LEUPEN
}

British Medical fournal, 1971, 1, 82-84

\section{Summary}

House dust from high mountainous areas of Switzerland contains very few house-dust mites. In contrast to lower-lying regions, only very small quantities of house-dust allergen are found at high altitudes.

The cause of this phenomenon seems likely to be the climatic conditions in the high mountains of Europe, where cold air leads to extremely low humidity levels indoors. The soil conditions and a type of construction providing good protection against the penetration of water also contribute to dry conditions in houses. These factors prevent the development of large populations of allergen-producing house-dust mites.

The beneficial effect of a stay at high altitudes on patients with atopic asthma is probably due to the low concentrations of house-dust allergen.

\footnotetext{
Department of Allergology, University Hospital, Leiden, The Netherlands

F. TH. M. SPIEKSMA, PH.D

M. J. LEUPEN, certified building engineer (Present address: Research Institute for Public Health Engineering, TNO, Delft, the Netherlands)

Netherlands Sanatorium, Davos, Switzerland

P. ZUIDEMA, M.D., Director
}

\section{Introduction}

For almost a hundred years physicians in Switzerland and elsewhere have noticed that some patients with asthma lose 0 their symptoms during a stay in high mountainous regions. $\vec{N}$ The beneficial effect has been ascribed to the thin air, low $\sigma$ oxygen pressure, solar radiation, temperature, and other often $\frac{D}{0}$ vaguely indicated properties of the mountainous air. Only after allergy had been recognized as an important aetiological $\tilde{N}$ factor in the development of asthma was any other explana- $\mathbb{W}$ tion of the favourable effect of the mountain climate seriously $\vec{\sigma}$ considered-namely, the absence of harmful substances 0 capable of causing attacks of asthma in persons oversensitive $\mathbb{\Phi}$ to them.

Storm van Leeuwen et al. (1924) studied the response of $\frac{T}{0}$ asthmatics to residence in mountainous areas and found that $\overrightarrow{\mathbb{D}}$ the improvement in the patients' condition started at an alti- $\frac{\mathcal{D}}{\mathbb{D}}$ tude of $1,200 \mathrm{~m}$ and became maximal above $1,600 \mathrm{~m}$. The $\frac{\Omega}{\sigma}$ beneficial effects were found, however, to be only temporary.

Recent work has done much to provide a scientific explana- $\frac{8}{0}$ tion for the success of high-altitude treatment of asthma. The discovery of the house-dust mite Dermatophagoides pteronys- $\frac{\bar{a}}{2}$ sinus as the producer of house-dust allergen (Voorhorst et al., 1964, 1967) and its prevalence in damp and moderately damp climates have suggested that it is the most important cause of allergic asthma in low-lying districts. At high altitudes the 